\title{
Molecule transfer mechanism in 2D heterostructured lamellar membranes: The effects of dissolution and diffusion
}

\author{
Chongchong Chen ${ }^{1}$, Xiaoli Wu ${ }^{1}$, Jie Zhang ${ }^{1}$, Jingjing Chen ${ }^{1}$, Xulin Cui ${ }^{1}$, Wenpeng Li $^{1}$, \\ Wenjia $\mathrm{Wu}^{1}$, and jingtao wang ${ }^{1}$ \\ ${ }^{1}$ Zhengzhou University
}

August 7, 2021

\begin{abstract}
Two-dimensional (2D) lamellar membranes are promising for efficient molecule transfer, while the underlying molecule transfer mechanism is rarely elucidated. Herein, heterostructured nanosheets are prepared by self-assembling small-sized hydrophilic cyanuric acid melamine $(\mathrm{CMN})$ and hydrophobic g-C3N4 nanosheets. The resultant lamellar membranes show comparable affinity to both polar and nonpolar solvents, allowing them to dissolve on membrane surface and diffuse through membrane channels. Permeance results demonstrate that the transfer of polar solvents is controlled by dissolution and diffusion processes, while that of nonpolar solvents is governed by dissolution process. And the corresponding equations are established. Importantly, polar solvents are induced to form ordered arrangement in hydrophilic nanodomains and then maintain this state in hydrophobic nanodomains, affording low-resistance transfer thus high permeance: $1025 \mathrm{~L} \mathrm{~m}-2 \mathrm{~h}-1$ bar-1 for acetonitrile. In contrast, nonpolar solvents with disordered arrangement acquire lower permeance than that of polar ones, but with comparable diffusion ability in these membranes.
\end{abstract}

\section{Hosted file}

Manuscript.pdf available at https://authorea.com/users/429521/articles/533211-moleculetransfer-mechanism-in-2d-heterostructured-lamellar-membranes-the-effects-of-dissolutionand-diffusion 